\title{
HI Gas in Disk and Dwarf Galaxies in the Semi-analytic Models of Galaxy Formation $\dagger$
}

\author{
Jian Fu ${ }^{1}$, Jing Wang ${ }^{2}$, Yu Luo ${ }^{3}$ \\ ${ }^{1}$ Key Laboratory for Research in Galaxies and Cosmology, Shanghai Astronomical \\ Observatory, CAS, 80 Nandan Road., Shanghai, 200030, China, \\ email: fujian@shao.ac.cn \\ ${ }^{2}$ CSIRO Astronomy and Space Science, PO box 76, Epping, NSW 1710, Australia \\ ${ }^{3}$ Purple Mountain Observatory, 2 West Beijing Road, Nanjing, 210008, China
}

\begin{abstract}
We construct the radially-resolved semi-analytic models of galaxy formation based on the L-Galaxies model framework, which include both atomic and molecular gas phase in ISM. The models run on the halo outputs of $\Lambda$ CDM cosmology N-body simulation. Our models can reproduce varies observations of $\mathrm{HI}$ gas in nearby galaxies, e.g. the HI mass function, the HI-to-star ratio vs stellar mass and stellar surface density, universal HI radial surface density profile in outer disks etc. We also give the physical origin of HI size-mass relation. Based on our model results for local dwarf galaxies, we show that the "missing satellite problem" also exists in the HI component, i.e., the models over-predict dwarf galaxies with low HI mass around the Milky Way. That is a shortcoming of current $\Lambda$ CDM cosmology framework. Future survey for HI gas in local dwarf galaxies (e.g. MeerKAT, SKA \& FAST) can help to verify the nature of dark matter (cold or warm).
\end{abstract}

Keywords. galaxies: formation, galaxies: ISM, ISM: atoms

\section{The Models}

This work is based on Munich semi-analytic model (L-Galaxies, Guo et al.2013), which describes the physical prescriptions of the baryonic matter processes in the galaxy formation and evolution, e.g reionization, gas cooling and infall, AGN and SN feedback, star formation and metal production, galaxy merger and star burst, ram pressure stripping, tidal disruption of satellites etc. The models run on the $\Lambda \mathrm{CDM}$ cosmology simulation outputs Millennium, Millennium II and Aquarius haloes. Our models develop a method to trace the radial profiles of star, gas, star formation and some other physical processes in each galaxy disk. We calculate the conversion fraction between atomic and molecular gas in ISM, and then adopt the molecular gas proportional star formation law in the model $\Sigma_{\mathrm{SFR}} \propto \Sigma_{\mathrm{H}_{2}}$ (See Fu et al.2013 for the model detail).

\section{Model Results}

Fig. 1 shows the stellar mass functions, molecular gas mass functions and atomic gas mass functions from the model results at $z=0$. The model can reproduce the galaxy mass functions from the observations in dwarf galaxies, disk galaxies and massive elliptical galaxies.

The left panel of Fig. 2 shows the size-mass relation of HI gas in the model galaxies and observations. Broeils \& Rhee (1997) first found that tight correlation exists between HI mass and HI disk size, e.g $M_{\mathrm{HI}} \propto R_{\mathrm{HI}}^{1.96}$, which means the average HI surface density in different galaxy disk are almost same. Based on our model processes, we found that the HI size-mass relation is caused by the atomic-molecular gas conversion and ionization of neutral gas, e.g HI gas in high surface density regions tends to form molecular gas and HI gas in low surface density region

$\dagger$ Supported by Natural Science Foundation of China (No.11303072, U1531123). 

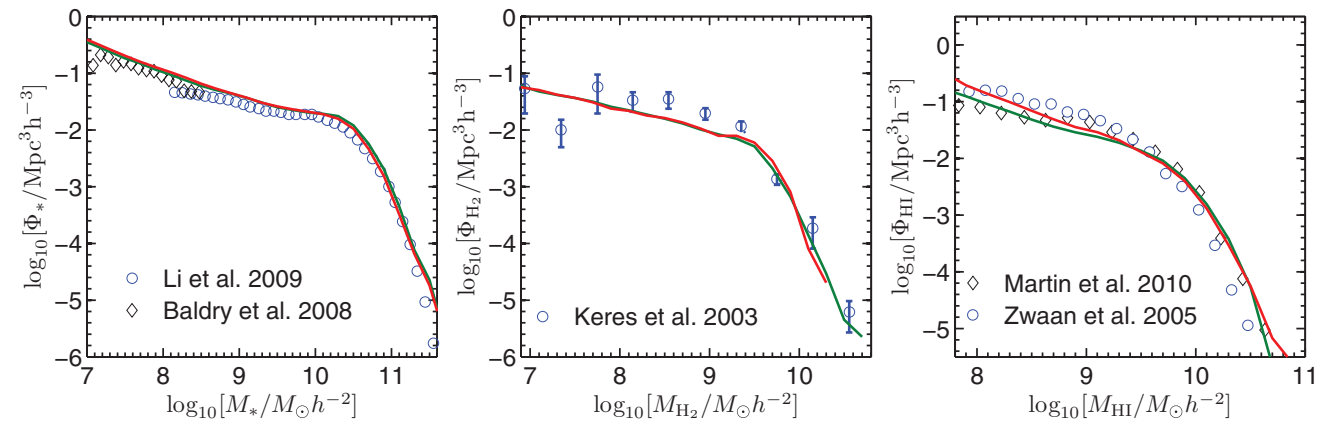

Figure 1. The galaxy mass functions of star, $\mathrm{H}_{2}$ and $\mathrm{HI}$ at $\mathrm{z}=0$. The green and red solid curves in each panels represent the model results, and the black and blue dots are from observations.
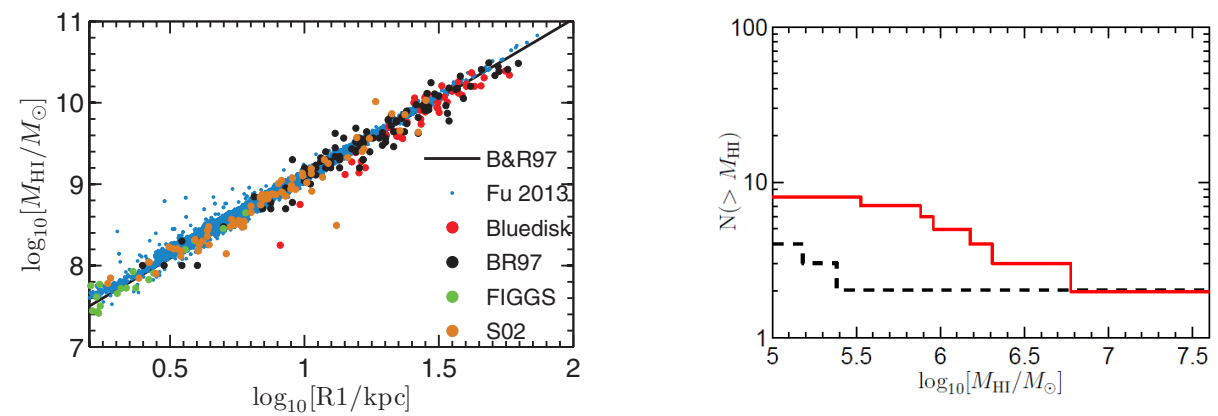

Figure 2. Left: The size-mass relation of HI gas in disk galaxies. The blue dots are from the model results at $z=0$; other dots are from various observations, and the black line are the fitting relation by Broeils \& Rhee (1997). Right: The cumulative number of satellite galaxies around the Milky Way vs HI mass. The red curve is the model results based on Aquarius halos, and the dashed curve is from the observation results by Grcevich \& Putman(2009).

tends to be ionized by UV background. The narrow distribution for the value of HI surface density leads to tight correlation between HI mass and HI disk size (Wang et al.2014).

The right panel of Fig. 2 shows the number count of dwarf satellite galaxies around the Milky Way in given HI mass bins. Comparing the model results based on Aquarius haloes (the solid curve) and the observations (the dashed curve from Grcevich \& Putman 2009), the model overpredicts the number of satellite galaxies with low HI gas mass, which is similar to the "missing satellite problem" for low luminosity satellite galaxies. This problem indicates the shortcomings in the baryonic physics in dwarf galaxies (e.g reionization, ram pressure stripping of cold gas, SN feedback in dwarf galaxies) or the nature of dark matter (WDM instead of CDM).

\section{References}

Broeils, A. H., \& Rhee, M.-H. 1997, A\&A, 324, 877

Fu, J., Kauffmann, G., Huang, M.-L., et al. 2013, MNRAS, 434, 1531

Grcevich, J., \& Putman, M. E. 2009, ApJ, 696, 385

Guo, Q., White, S., Angulo, R. E., et al. 2013, MNRAS, 428, 1351

Wang, J., Fu, J., Aumer, M., et al. 2014, MNRAS, 441, 2159 